\title{
Preliminary design of FTS-2: an imaging Fourier transform spectrometer for SCUBA-2
}

\author{
David A. Naylor*a, Brad G. Gom ${ }^{\mathrm{a}}$, Baoshe Zhang ${ }^{\mathrm{a}}$ \\ ${ }^{a}$ Dept. of Physics, University of Lethbridge, 4401 University Drive, Lethbridge, Alberta, Canada \\ T1K $3 \mathrm{M} 4$
}

\begin{abstract}
We present the preliminary design of FTS-2, an imaging Fourier transform spectrometer (IFTS) for use with SCUBA-2, the second generation, wide-field, submillimetre camera currently under development for the James Clerk Maxwell Telescope (JCMT). This system, which is planned for operation at the start of 2007, will provide simultaneous broadband spectral imaging across both the 850 and $450 \mu \mathrm{m}$ bands with variable resolution ranging from resolving powers of $\mathrm{R} \sim 10$ to 5000 . The spectrometer uses a folded Mach-Zehnder configuration and novel intensity beam dividers. The mechanical and optical design of FTS-2 as of the Critical Design Review stage of the project are discussed, along with the interfaces with SCUBA-2 and the JCMT.
\end{abstract}

Keywords: Fourier, Spectrometer, SCUBA-2, Submillimetre, JCMT

\section{INTRODUCTION}

One of the major unsolved problems of modern astrophysics is a detailed understanding of the processes underlying star formation. Since star formation is intimately linked with planetary formation, this problem has fundamental significance not only for our immediate galactic environment, but also in the study of the early universe. Despite significant progress in the past two decades ${ }^{1,2}$, several aspects of star formation remain poorly understood, including:

- What are the physical conditions at the onset of, and what initiates protostellar collapse in a molecular cloud?

- What is the efficiency and time scale of star formation?

- What determines the distribution of stellar masses at birth (the initial mass function)?

It is now well established that stars form from the collapse of dense cloud cores in the interstellar medium, but remain cocooned in their natal envelopes of gas and dust. The optical opacity of this material is so great that these cores can only be studied at far-infrared and submillimetre wavelengths where the dust emission is optically thin. A number of space and ground-based astronomical instruments, many of them discussed in these proceedings, are currently under development to address these questions.

Building on the highly successful SCUBA camera ${ }^{3}$, which operated on the James Clerk Maxwell Telescope (JCMT) between 1997 and 2005, a new, large format, submillimetre camera, SCUBA-2, is currently under development for use at the JCMT ${ }^{4}$. SCUBA-2 features two dc-coupled, monolithic TES filled arrays with a total of $\sim 10,000$ bolometers, unlike previous detectors which have used much smaller arrays of discrete bolometers. With its larger format and increased sensitivity, SCUBA-2 promises a factor of 1000 increase in mapping speed compared to its predecessor.

While SCUBA-2 will provide unprecedented morphological information on the structure of submillimetre astronomical sources, their composition and physical conditions can only be determined through imaging spectral measurements. A Fourier Transform Spectrometer (FTS) has been selected as the optimal instrument for medium resolution spectroscopy when used in conjunction with SCUBA-2. This choice was based on the well known advantages of Fourier spectroscopy, including:

*naylor@uleth.ca; phone 1403 329-2426; fax 1403 329-2057; www.uleth.ca/phy/naylor/ 
- Simultaneous broadband, readily adjustable intermediate resolution measurements across both the 850 and 450 $\mu \mathrm{m}$ SCUBA-2 bands

- The best instrumental line shape function of any spectrometer

- Intrinsic wavelength calibration and relatively easy intensity calibration

FTS-2 will be primarily a galactic spectrometer (e.g. spectral index mapping of molecular clouds), but will also provide useful information on bright nearby galaxies and planetary atmospheres. FTS-2 thus fills a niche between the two band SCUBA-2 continuum images and the higher spectral resolution, but limited-sized images produced by the JCMT heterodyne facility instrument HARP-B.

Since the layout of the JCMT - SCUBA-2 feed optics was well advanced prior to the decision to include an intermediate resolution spectrometer, the mechanical, optical, and software design of FTS-2 was significantly more challenging. Previous papers have discussed the conceptual design of FTS-2. ${ }^{5,7}$ In this paper we review the current PDR level design of the FTS-2 instrument emphasizing the optical, mechanical and software design, as the project enters the CDR phase.

\section{OPTICAL DESIGN}

FTS-2 intercepts the SCUBA-2 optical beam near an intermediate image plane directly outside the telescope elevation bearing opening (see Fig. 1). The optical design problem is essentially to reproduce the original image and pupil after the beam for each port has passed through the interferometer, while maintaining unity image magnification, in order to allow the instrument to be used with the existing SCUBA-2 feed optics. Within the interferometer, there are additional design constraints that the beams at the rooftop mirrors must be collimated, there must be pupils located at the rooftop mirrors (at the ZPD location) for symmetry, and there must also be pupils near the beamsplitters in order to minimize the beamsplitter diameters.

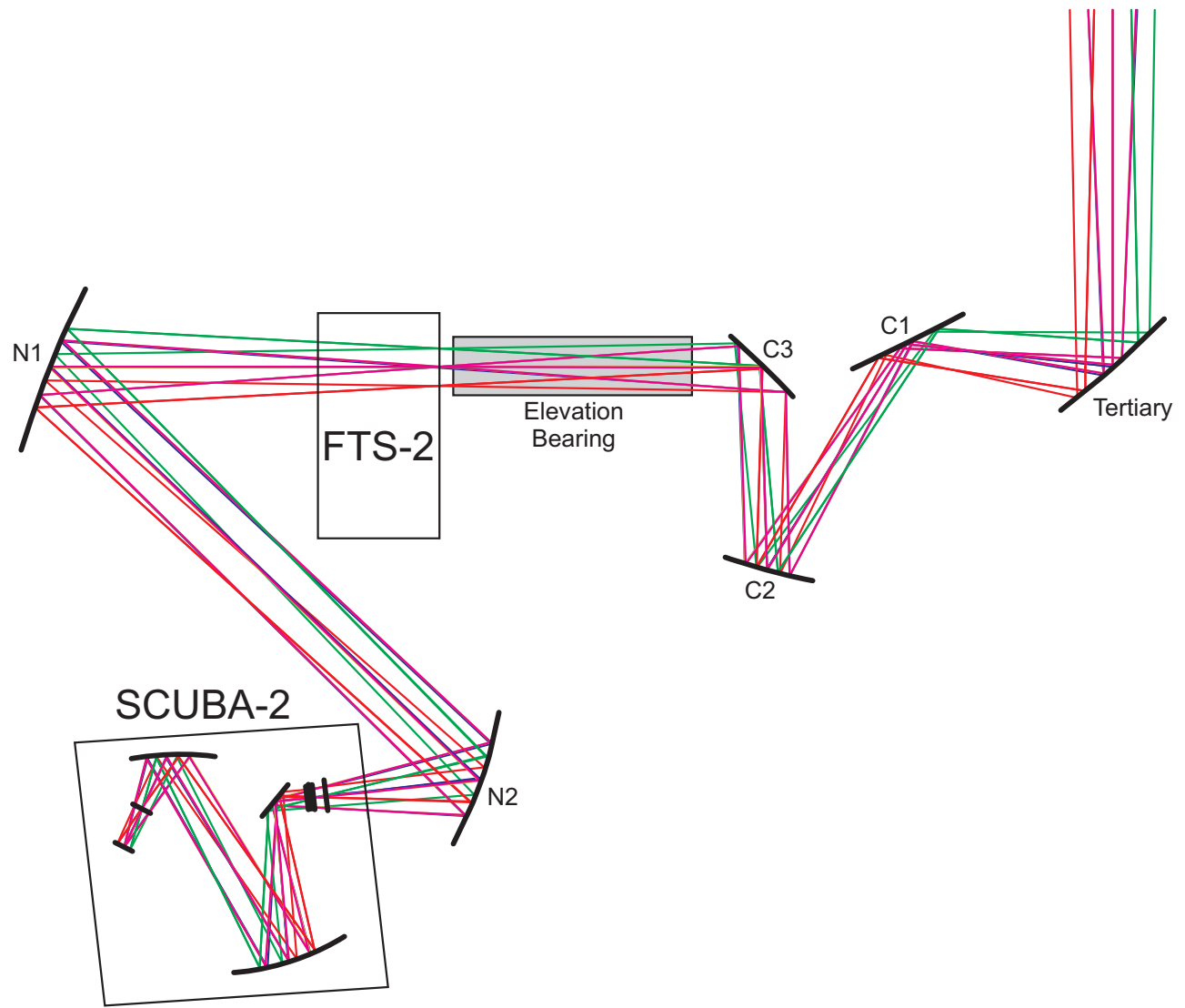

Fig. 1. FTS-2 location within the JCMT SCUBA-2 feed optics. 
FTS-2 uses a dual-input, dual-output Mach-Zehnder interferometer configuration ${ }^{6}$ which allows 2 ports to be placed on the sky for atmospheric cancellation. A linear schematic of the FTS-2 optics for one port of the interferometer is shown in Fig. 2. A pickoff mirror intercepts one quadrant of the SCUBA-2 FOV near an intermediate image plane outside the telescope elevation bearing. Mirror FM2_1 folds the beam downwards and creates an image after the first beamsplitter (BS), minimizing the beamsplitter diameter. Mirror FM1_3 folds the beam at the breadboard level and forms a pupil image at the apex of the moving rooftop mirrors (RT) when the interferometer is at ZPD. After reflection from the RT mirrors, fold mirrors FM1_4 and FM2_2 return the beam to the elevation bearing level and reproduce the input pupil and image at the required positions before the return mirror feeds the beam back to the SCUBA-2 feed optics at N1.

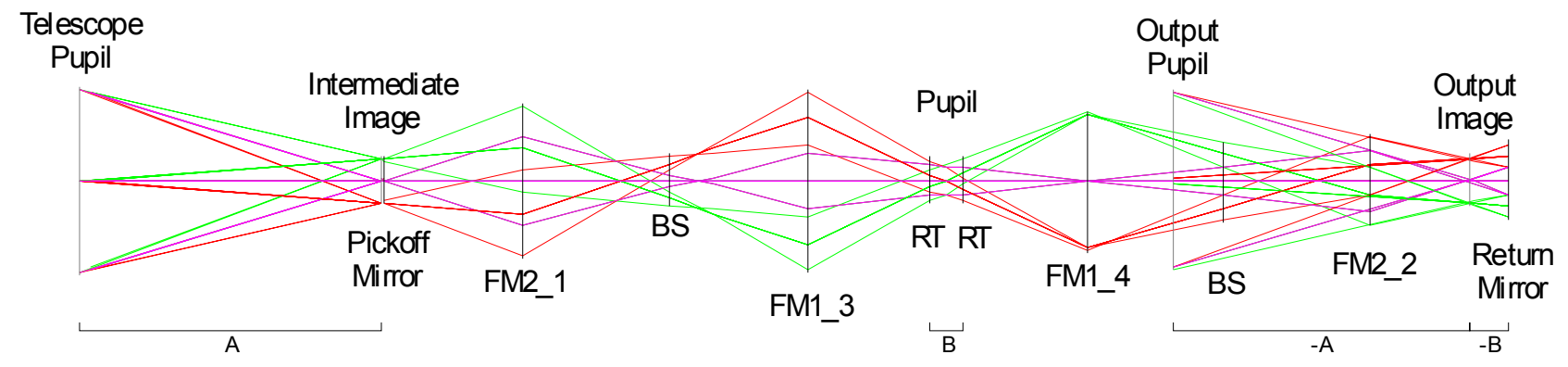

Fig. 2. Simplified optical schematic of FTS-2 for one port (not to scale). Beam diameters at the beamsplitters (BS) are kept as small as possible. The sizes and positions of the telescope pupil and image at the telescope elevation bearing are reproduced at the output of the FTS, taking into account the thickness of the system (B).

Since the JCMT optical and structural framework designs are already fixed, the FTS-2 optical and mechanical designs are highly interdependent and tightly constrained. In order to fit the optics in the available space, the interferometer design is folded vertically ${ }^{7}$ as shown in Fig. 3.

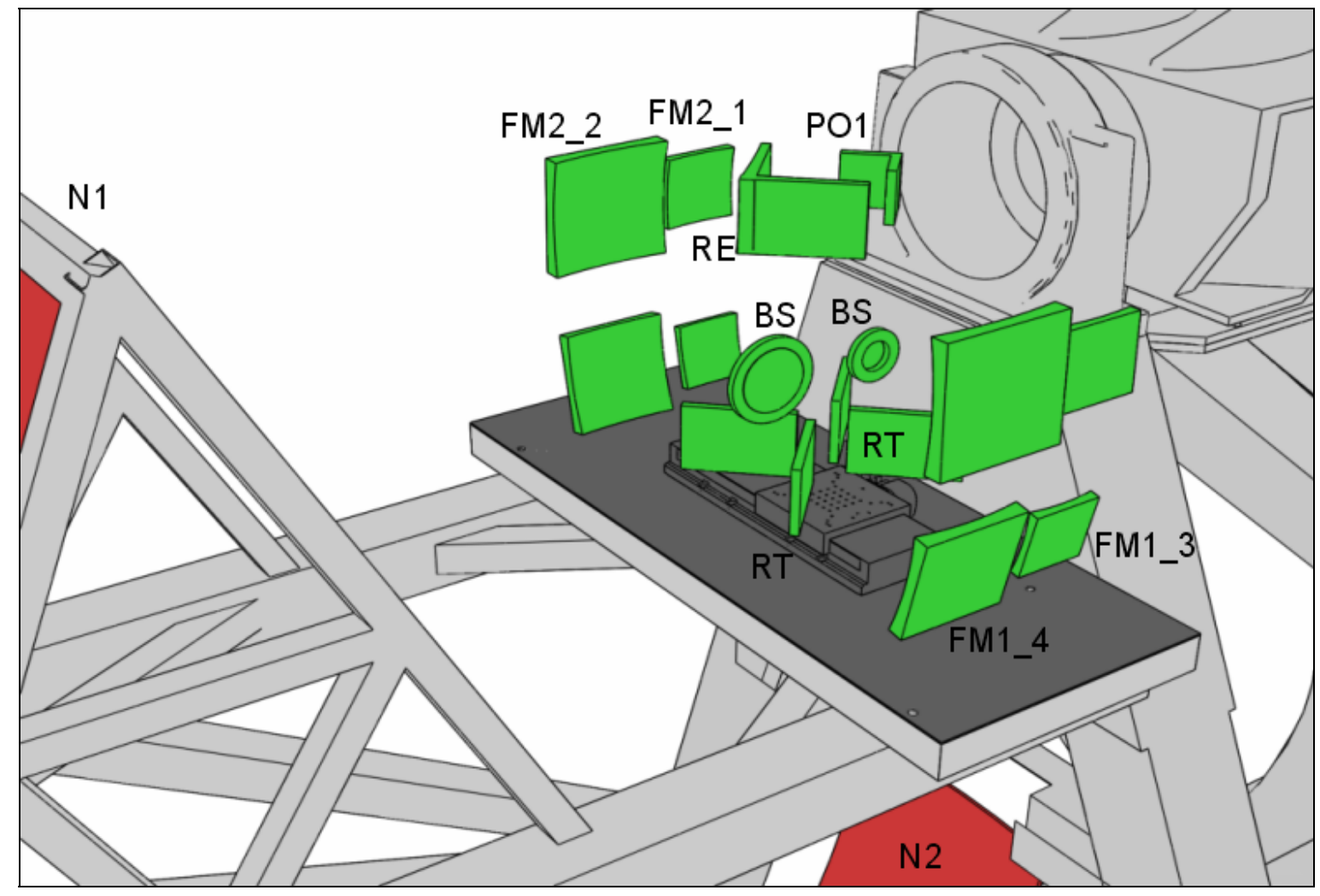

Fig. 3. Folded optical layout of FTS-2 at the mounting location on the SCUBA-2 N1 mirror support framework. The FTS-2 mechanical framework is hidden for clarity. 
It is impossible with the existing SCUBA-2 feed optics to achieve the spectral resolution design goal over the entire SCUBA-2 field of view and optimizing the resulting tradeoff between FOV and spectral resolution within the constraints imposed by the fixed space envelope has proven to be a challenge.

Optimization of the optical design was done in Zemax taking into account the physical constraints of the available space envelope. With the constraints mentioned above and the limited mounting space, the maximum FOV is ultimately limited by the maximum practical mirror diameters (roughly $400 \mathrm{~mm}$ diameter) to approximately $5 \mathrm{arcmin}^{2}$.

\section{MECHANICAL DESIGN}

Mounting the 16 FTS-2 mirrors in a compact folded configuration without creating any mechanical or optical interference with the existing JCMT and SCUBA-2 systems was a major challenge in the mechanical design. Not all the optical components could be mounted directly to the optical breadboard, so a framework design was adopted to support the upper optics as well as allow the pickoff and return mirrors to be retracted from the SCUBA-2 beam when not in use. A Newport damped optical breadboard forms the base of the system and provides isolation from telescope vibration, as well as a flat mounting surface for all of the FTS-2 hardware. The breadboard will be supported by seven adjustable feet when mounted on the JCMT N1 support framework.

\subsection{Mechanical framework}

A tubular 6061-T6 aluminum framework will support all of the FTS-2 hardware which is not on the breadboard level, including the beamsplitters, upper fixed mirrors, pickoff and return mirrors, and the retraction assembly. The framework will be bolted to the optical breadboard and protective covers will be fitted to the outside to prevent damage and dust accumulation. The asymmetrical design of the framework avoids interference with the SCUBA-2 beam and the JCMT primary mirror backing structure while maintaining high rigidity. The framework sub-assemblies will be welded separately and then bolted together, in order to facilitate shipping of the instrument. Since the framework will measure roughly $2 \mathrm{~m} \times 0.6 \mathrm{~m} \times 1.3 \mathrm{~m}$, it will be disassembled into several sections which can be packaged flat for shipping to the JCMT. The framework is shown in Fig. 4.

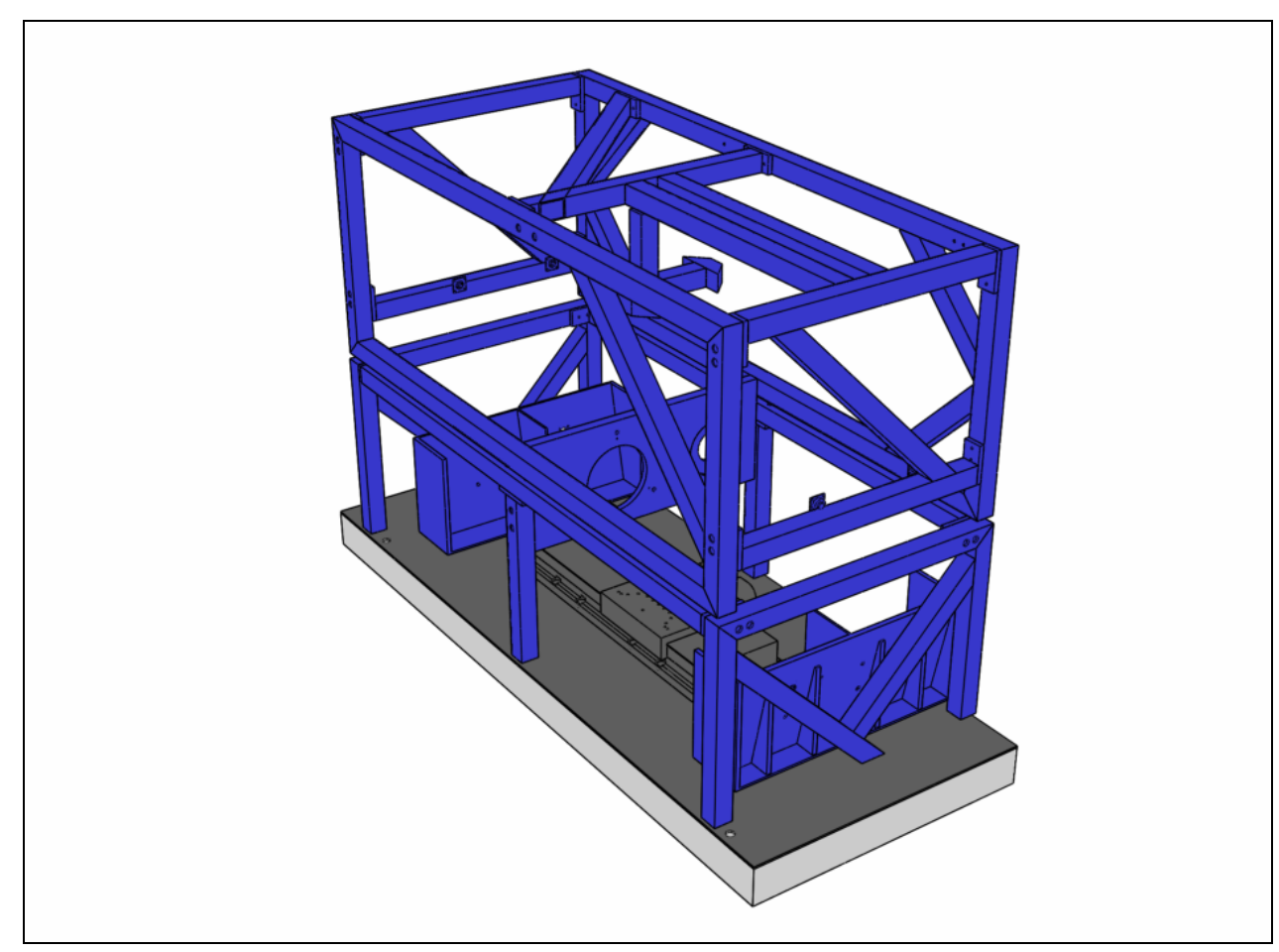

Fig. 4. FTS-2 mechanical framework mounted on the optical breadboard. Optics are hidden for clarity. 


\subsection{Mirror mounts}

Maximizing the FTS-2 field of view requires mirrors with diameters up to $400 \mathrm{~mm}$ within the interferometer. The mass for each of these mirrors is expected to be in the range of three to six kilograms. The mirror mounting features must be compact due to the proximity of the mirrors to each other, particularly the pickoff and return mirrors and the rooftop mirrors. As there are no suitable commercially available mirror mounts, the FTS-2 mirror mounts were custom designed.

The mirror mount design incorporates a spherical roller bearing for a pivot. A roller bearing was chosen over a plain spherical bearing for the reduced friction, which allows for smoother adjustment. The spherical bearing is fastened into a bracket plate, with the centre of the mirror hanging from a bolt passing through the bearing. If required, a third axis of adjustment (translation) can be obtained by inserting shims in between the bolt and the mirror. The bracket plate also houses the two adjusters necessary for the elevation and azimuth adjustment of the mirror, and a tensile spring which ensures the stiffness of the assembly and forces the mirror to return to its position following a shock.

To prevent the adjuster tips from scarring the aluminum mirror, two steel inserts are integrated into the mirror. The elevation insert has a V-groove to prevent rotation of the mirror about the axis of the spherical bearing. The elevation adjuster was chosen for this function as the moment induced by the mirror's weight acts on it and thus it will provide more stability. The machining of the bracket plate is minimal, making this design a very cost-effective solution. Fig. 5 depicts an assembled mirror mount featuring two Zaber Technologies ${ }^{8}$ NA11-16B motorized adjusters and a compression spring assembly.

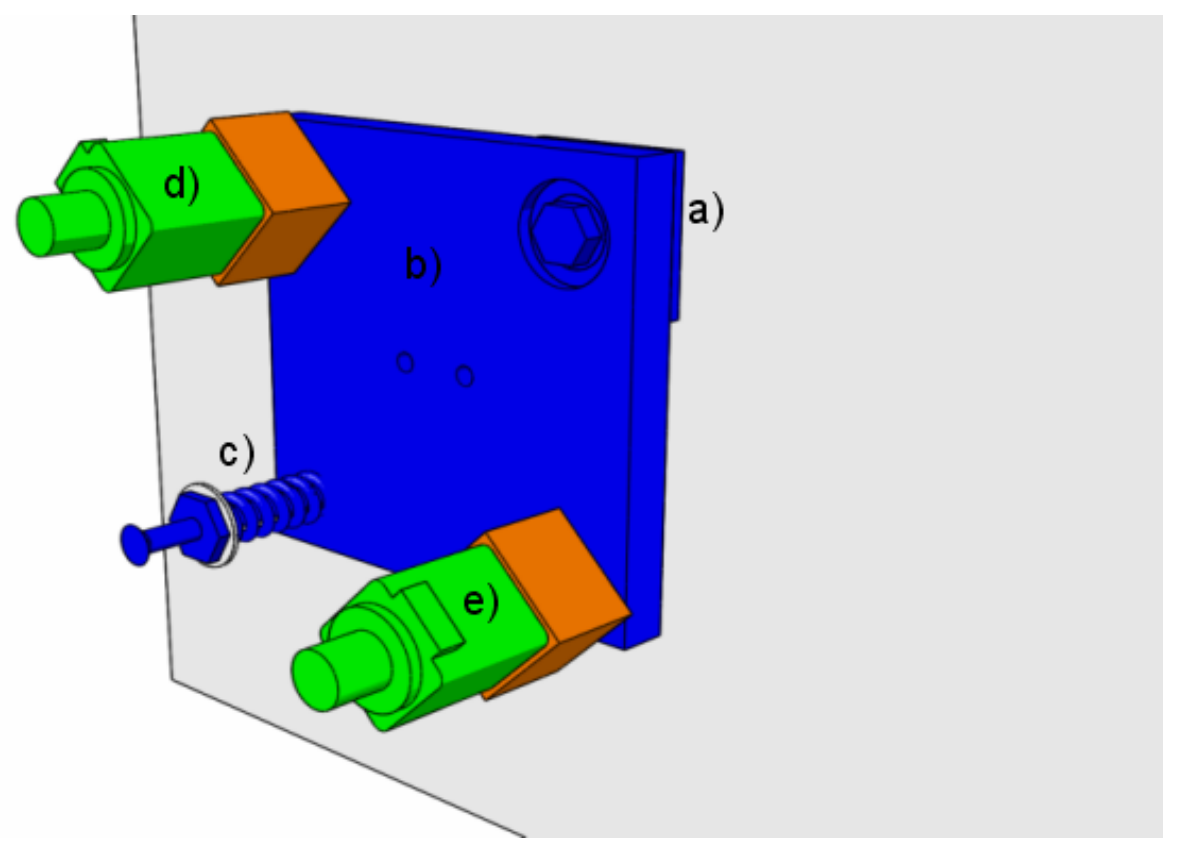

Fig. 5. Spherical bearing mirror mount, showing a) spherical bearing, b) bracket plate, c) tensioning spring, d) and e) motorized adjusters.

The spherical bearings allow an $8^{\circ}$ range of adjustment in each axis. The static and dynamic loading are well below the ratings for the bearings, rendering the bearings maintenance free. Testing in the lab using a small telescope ${ }^{9}$ has demonstrated the resolution to be on the order of 0.1 arc seconds per actuator microstep. The adjustment remains very smooth and predictable at the arc second level, and the mirror returns to its desired position when subjected to normal handling shock. This design could be easily adapted to other applications requiring large mirrors.

\subsection{Linear translation stage}

The FTS-2 rooftop mirrors are displaced by an Aerotech ALS5000 series linear motor translation stage ${ }^{10}$ with $450 \mathrm{~mm}$ of travel. The brushless, non-contact linear servo motor features zero backlash and the stage metrology is based on a noncontact Heidenhain linear encoder. The stage has integral limit transducers to prevent driving the moving platform beyond the end of travel. Accelerations of $30 \mathrm{~m} / \mathrm{s}^{2}$ and velocities of $2 \mathrm{~m} / \mathrm{s}$ can be achieved with a maximum permissible load of $135 \mathrm{~kg}$, easily accommodating the motion profiles for the FTS-2 rooftop mirror masses. 


\subsection{Rooftop mirror assembly}

The rooftop mirrors sit atop the Aerotech translation stage moving platform, which moves to provide varying optical path difference between the interferometer beams. Each rooftop mirror sub-assembly supports one mirror from each side of the interferometer. This allows one set of mirrors to be removed for alignment of the remaining interferometer mirrors and then reinstalled with very little disturbance to their alignment.

\subsection{Retraction mechanism}

It is necessary for the FTS-2 pickoff and return mirrors to be retracted from the SCUBA-2 beam when FTS-2 is not in operation. These mirrors also require remote actuators for periodic adjustment of the alignment to the SCUBA- 2 beam. The pickoff mirror retraction assembly consists of a rail and carriage system, a ball screw, and a Zaber Technologies stepper motor. The ball screw and stepper motor combination will allow the mirrors to be positioned in the beam with a resolution of $0.1 \mu \mathrm{m}$. The rail will be bolted to the FTS-2 framework at a spacing of $60 \mathrm{~mm}$ to minimize deflection and produce superior repeatability. The Zaber motor will be driven in a step-back fashion to compensate for backlash such that it will always approach its destination from the same direction.

\section{OBSERVING MODES}

Atmospheric emission is the dominant source of radiant loading at submillimetre wavelengths. Moreover, variations in atmospheric emission are particularly problematic for an FTS where, upon Fourier transformation, they introduce spectral features into the resulting spectrum. By utilizing the second input port of the Mach-Zehnder FTS to view an adjacent background sky position, variations in atmospheric emission can, to first order, be cancelled by the subtractive properties of the FTS. Moreover, this cancellation results in a dramatic reduction in the dynamic range required in the resulting interferogram. Also, the dual output ports of the FTS provide complementary data which can further reject common mode noise present in the often hostile telescope environment, resulting in a factor of $\sqrt{ } 2$ increase in $\mathrm{S} / \mathrm{N}$. Proper selection of a background location is crucial to the success of this technique; the location of the second input port must be carefully considered in the observation planning.

Using dual input ports, the moving mirror may be scanned continuously (Rapid-Scan or RS) or stepped discretely (Stepand-Integrate or SI). With RS, the resulting interferograms will not be sampled uniformly in optical retardation since the SCUBA-2 data acquisition system is independent of the FTS-2 scanning mechanism. This will necessitate the use of a non-uniform FFT or an interpolation process in the processing pipeline. Algorithms to cope with this problem have already been developed for the SPIRE spectrometer ${ }^{11}$ by members of our group.

We are also investigating the potential use of the step-and-integrate operating mode to improve observational efficiency and atmospheric noise rejection. In this mode, the optical path difference in the interferometer is incremented in discrete steps and data is read out only when the mirrors are stationary, thereby ensuring that the interferogram is sampled on a uniform position grid. This mode could in principle be used with the single-port mode in conjunction with the SCUBA-2 DREAM mode ${ }^{12}$, however, the baseline plan is to use the Rapid Scan dual-port atmospheric cancellation technique.

\subsection{Undersampling}

Since the SCUBA-2 filters will have extremely high out-of-band rejection, the interferograms may be sampled sparsely and the resulting aliasing of the spectra can be easily removed. This will allow high resolution spectra to be obtained in shorter scan times, which will reduce the effects of sky rotation and atmospheric noise. By proper selection of the optical path sampling interval, both the 450 and $850 \mu \mathrm{m}$ bands can be aliased simultaneously without any loss of information within the bands.

Normal Nyquist sampling requires that the interferograms be sampled every $0.02 \mathrm{~cm}$ of OPD, but this interval can be increased to $0.1 \mathrm{~cm}$ through the use of undersampling, which translates to a factor of 5 increase in acquisition speed. We have tested this technique with the U of L FTS ${ }^{13}$ using both the normal rapid-scan and the step-and-integrate modes ${ }^{7}$.

\subsection{Resolution}

The FTS- 2 instrument has a continuously variable resolution ranging from $\Delta v \sim 0.5$ to $0.006 \mathrm{~cm}^{-1}$. (The translation stage will allow slightly better resolution if some vignetting of the outer pixels can be tolerated.) While the FTS-2 resolution can be adjusted arbitrarily over the full range, the baseline plan is to provide only two resolution modes. The maximum resolution will be used for spectral line studies, while a lower resolution of $\sim 0.1 \mathrm{~cm}^{-1}$ will be used for Spectral Energy 
Distribution (SED) measurements ${ }^{7}$. With the fixed detector frame rate, higher resolution comes at the expense of longer acquisition times.

\subsection{Baseline Observing Modes}

FTS-2 observations can be classified as either SED measurements or spectral line studies. SED measurements only require a few spectral bins across a filter bandpass in order to characterize the continuum curvature, and can be accomplished with low resolution $\left(\sim 0.1 \mathrm{~cm}^{-1}\right)$ scans. On the other hand, spectral line studies require the maximum possible resolution. By grouping all observations into these two resolution categories, the processing and observation planning can be simplified.

In the low-resolution SED mode, scans can be double-sided without seriously affecting observing efficiency. With fully double-sided scans, the phase correction processing step is simplified greatly. The baseline plan is to implement the SED mode using the dual-port configuration to provide atmospheric correction. The nominal operating mode will be RS; the aliased SI mode combined with DREAM will be investigated during commissioning.

In the high-resolution spectral line mode, scans must be single sided to maximize the use of the linear stage travel and minimize the scan acquisition time. A short double-sided scan will provide phase information for the phase correction algorithm. The dual-port configuration will be used to provide atmospheric correction.

For dual-band operation, the acquisition time is limited by the $25 \mathrm{~cm}^{-1}$ Nyquist frequency for the $450 \mu \mathrm{m}$ band. If only the $850 \mu \mathrm{m}$ band is needed, then the acquisition time (and cube size) is reduced due to the lower $15 \mathrm{~cm}^{-1}$ Nyquist frequency. A summary of the acquisition times and interferogram lengths is given in Table 1.

Table 1. Scan parameters for the SED and Spectral Line modes.

\begin{tabular}{|r|c|c|c|c|}
\hline \multirow{2}{*}{} & \multicolumn{2}{|c|}{ SED } & \multicolumn{2}{c|}{ Spectral Line } \\
\cline { 2 - 5 } & Dual Band $850 \mu \mathrm{m}$ & Dual Band & $850 \mu \mathrm{m}$ \\
\hline Resolution & \multicolumn{2}{|c|}{$\sim 0.1 \mathrm{~cm}^{-1}$} & \multicolumn{2}{c|}{$\sim 0.006 \mathrm{~cm}^{-1}$} \\
\hline Scan type & \multicolumn{2}{|c|}{ Double-sided } & \multicolumn{2}{c|}{ Single-sided } \\
\hline Phase correction & \multicolumn{2}{|c|}{ Simple } & \multicolumn{2}{c|}{ Full } \\
\hline Scan mode & \multicolumn{2}{|c|}{ RS (SI) } & \multicolumn{2}{c|}{$100+6$} \\
\hline Total Travel (cm OPD) & \multicolumn{2}{|c|}{$6+6$} & 4 & 6.6 \\
\hline Velocity (cm/s OPD) & 4 & 1.8 & 26.5 & 15.9 \\
\hline Scan time (s) & 3 & 363 & 5300 & 3180 \\
\hline Frames & 605 & & & \\
\hline
\end{tabular}

\subsection{Mapping}

Mapping with FTS-2 will be complicated by vignetting effects and calibration issues with off-axis pixels. While correction of these effects should be possible after commissioning tests, mapping of extended regions will require postprocessing of the spectral data by the observer.

\section{SOFTWARE}

As an ancillary instrument, FTS-2 must be delivered with control and data reduction software which interfaces with the existing SCUBA-2 and JCMT systems. Data reduction code based on the SPIRE system ${ }^{11}$ has been developed for FTS-2, and custom control software has been written to control the translation stage and actuators.

\subsection{Data reduction pipeline}

Data reduction for SCUBA-2 and its ancillary instruments FTS-2 and POL-2 will be automated by the use of a data reduction pipeline. The main SCUBA-2 pipeline ${ }^{14}$ is written in object-oriented Perl. The pipeline parses 'recipes' and calls appropriate 'primitive' functions in various algorithm 'engines'. Since the implementation of the algorithm engines is independent of the pipeline itself, the FTS-2 algorithm engine can be written in Java to exploit existing SPIRE code. 
The Java FTS-2 data reduction algorithm engine consists of two major parts: an interface layer and a core layer. The interface layer provides a message interface for the SCUBA-2 data reduction pipeline to invoke the functions of the core layer. Both DRAMA ${ }^{15}$ and SOAP messaging interfaces are supported. The core layer is made up of five independent modules: I/O, Interpolation, Phase Correction, FFT, and the Quick Look (QL) display system.

The FTS-2 algorithm engine uses a multi-tier structure (see Fig. 6) consisting of three separate modules (referred to as 'actions' for DRAMA, or 'operations' for SOAP):

- set_parameters set the data reduction parameters

- data_reduction perform the core numerical computation of FTS-2 data reduction

- $\quad$ exit stop FTS-2 Engine and exit

From the perspective of the Pipeline, each action or operation has a corresponding primitive.

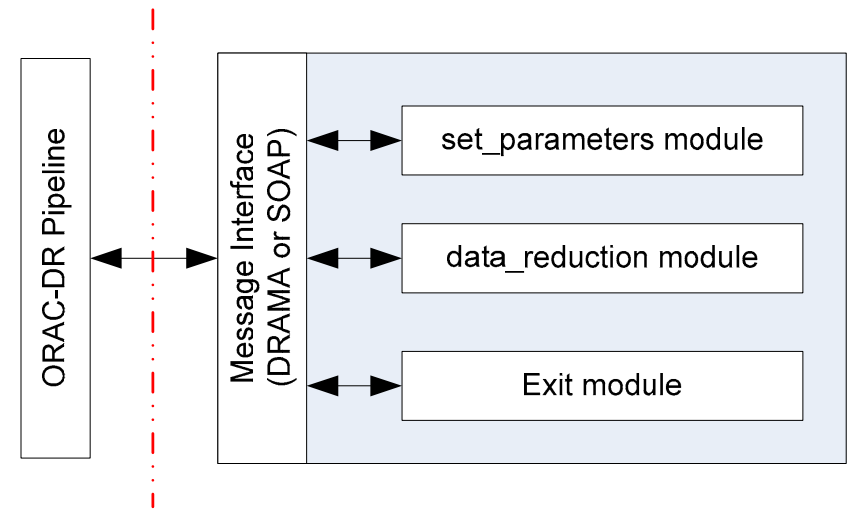

Fig. 6. FTS-2 algorithm engine messaging overview.

The SCUBA-2 DR pipeline calls the appropriate algorithm engine for the current instrument when the pipeline detects a new data file either from the Data Acquisition System (on-line mode) or from the data archive (off-line mode). After the algorithm engine completes the relevant tasks or exits, the SCUBA-2 data reduction pipeline regains control of the data flow. Fig. 7 shows a schematic of the FTS-2 data reduction module, which reduces raw interferogram data from the SCUBA-2 pipeline into spectral data files as well as reduced data for the SCUBA-2 Quick Look display system.

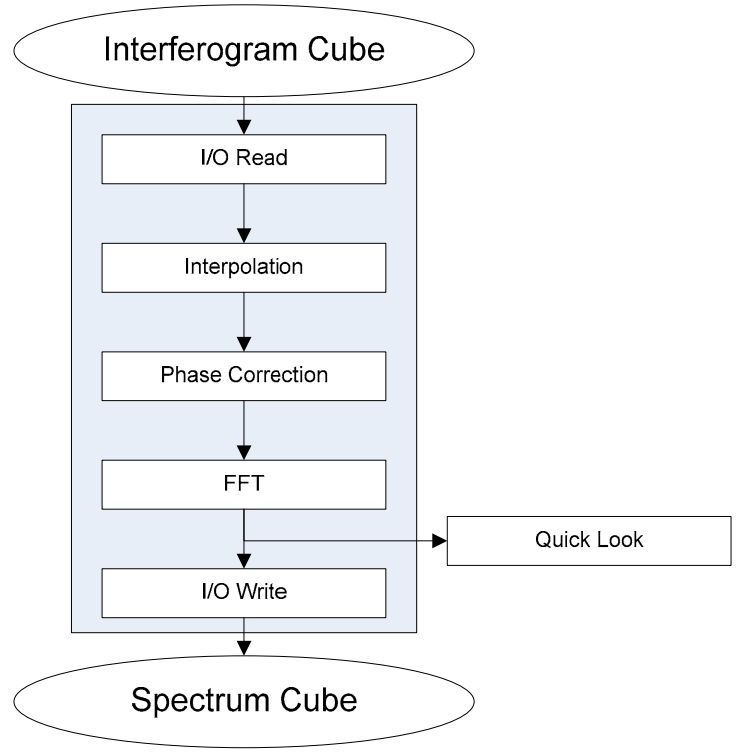

Fig. 7. FTS-2 data reduction engine modules. 
In order to separate the core numeric processing from both the data access layer and the message processing layer, the FTS-2 data reduction module is implemented as a Java class: ca.uol.aig.fts.drpipeline.DRPipeline. This class integrates I/O, Interpolation, Phase Correction, FFT and Quick Look into a pipeline to fully process FTS-2 interferogram data.

The FTS-2 engine I/O function of is implemented by Java class ca.uol.aig.fts.io.NDFIO, the interpolation function is implemented in the Java class ca.uol.aig.fts.fitting.CubicSpline, the phase correction function is implemented in the Java class ca.uol.aig.fts.phasecorrection.PhaseCorrection, and FFT processing is implemented in the classes RealDoubleFFT, RealDoubleFFT_Even, and RealDoubleFFT_Odd of the Java package ca.uol.aig.fftpack.

The FTS-2 engine uses four external Java libraries: a Java version of FFTPack ${ }^{17}$, Jama ${ }^{18}$, StarJava ${ }^{19}$, and a Java version of DRAMA. The Java version of FFTPack has been translated from the original FORTRAN code by the FTS-2 group. FFTPack is a well-known package of FORTRAN subprograms for the fast Fourier transform of periodic and other symmetric sequences, including complex, real, sine, cosine, and quarter-wave transforms. JAMA is a basic linear algebra package for Java developed by NIST. Two other required Java libraries are StarJava and DJAVA (Java version of DRAMA).

\subsection{Control systems}

Coordination and synchronization of the JCMT instruments, Observatory Control System ${ }^{20}$ (OCS) and Data Acquisition System are performed by the JCMT Real Time Sequencer ${ }^{21}$ (RTS). The FTS-2 RTS client will receive commands from the OCS and synchronizes their execution with the RTS master clock. In this way, the motion of the FTS mechanisms can be controlled by the automated OCS and the interferometer optical path difference can be read out synchronously with the $200 \mathrm{~Hz}$ SCUBA-2 frames. The JCMT uses a generalized software interface for all RTS compliant instruments, which significantly simplifies the control software development for the FTS-2 system. The FTS-2 RTS client software will use the $\mathrm{RTAI}^{22}$ real-time Linux operating system.

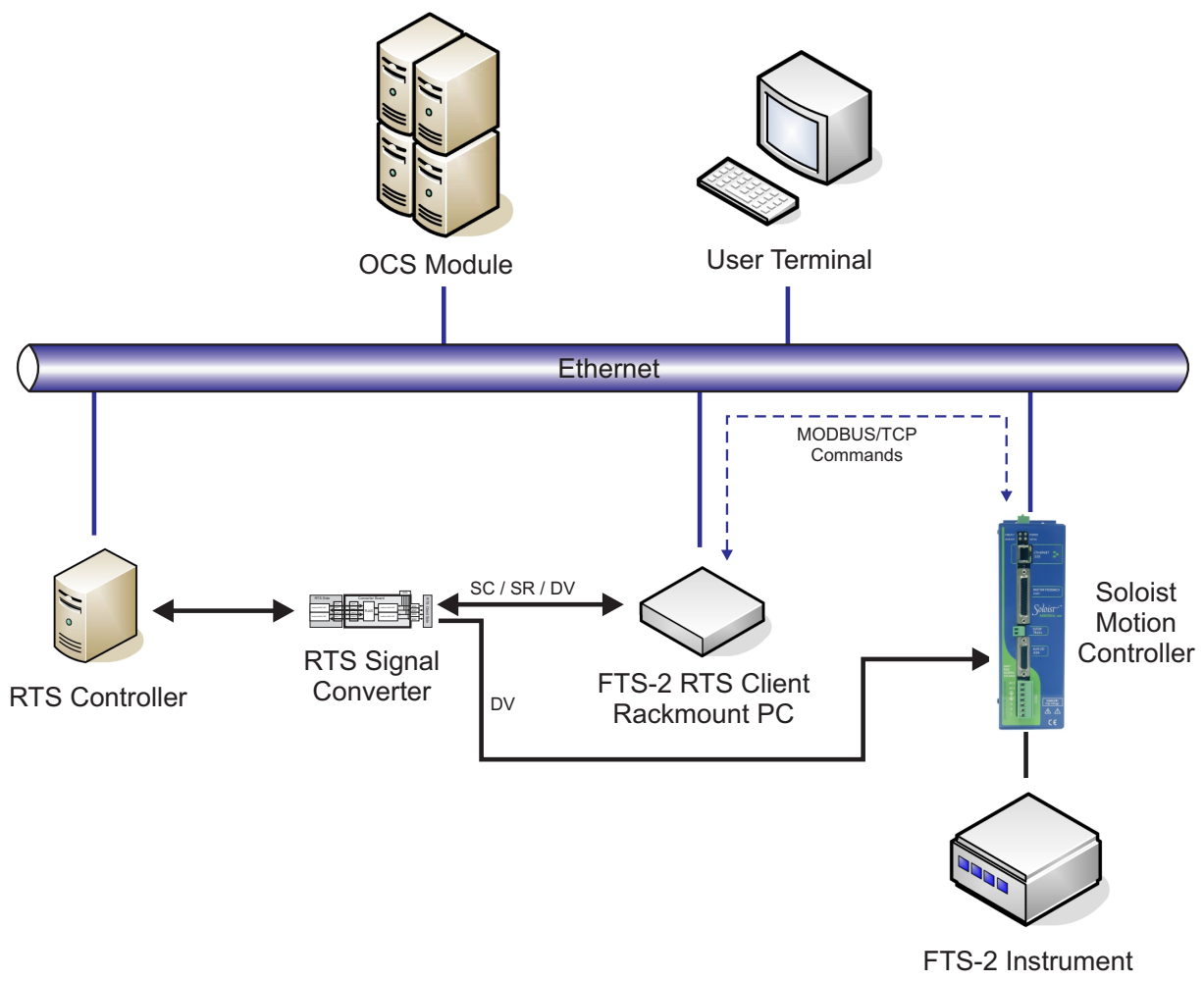

Fig. 8. Schematic of the FTS-2 control system interfaces with the JCMT OCS and RTS systems. 


\subsection{Data rate}

Data rates for the FTS-2 instrument will not exceed those for normal SCUBA-2 observations. In the RS mode, frames will be stored at $200 \mathrm{~Hz}$ before being reduced by the pipeline. Depending on the spectral range in the stored spectral cubes, the maximum reduced data volume before averaging will range from 0.25 to 0.5 times the raw data volume.

\subsection{Processing speed}

The FTS-2 engine was benchmarked on a Linux Fedora platform with a $2.8 \mathrm{Ghz}$ P4 CPU and 1 GB RAM. The benchmarks do not include any time that the ORAC-DR pipeline would take to call the algorithm engine. The phase correction function (PCF) decays very quickly at frequencies far from DC. Larger PCF lengths produce more accurate results, but require more computation time. Usually, in order to reduce the calculation time, the PCF is kept as short as possible. In the case of FTS-2, the PCF size never needs to be larger than 160 points. Benchmark results for the worstcase scenario (160 point PCF) are given in Table 2, showing that real-time processing should be possible in all observing modes.

Table 2. Processing benchmarks

\begin{tabular}{|c|c|c|c|c|c|c|c|c|c|}
\hline \multirow{2}{*}{ Scan Mode } & \multicolumn{3}{|c|}{$\begin{array}{c}\text { Interferogram Length } \\
\text { (points) }\end{array}$} & \multicolumn{4}{|c|}{ Benchmarks (s) } & \multicolumn{2}{|c|}{ Total Time (s) } \\
\hline & $\begin{array}{l}\text { Short } \\
\text { Wing }\end{array}$ & $\begin{array}{l}\text { Long } \\
\text { Wing }\end{array}$ & Total & $\mathbf{I} / \mathbf{O}(\mathbf{R} / \mathbf{W})$ & $\begin{array}{c}\text { Interpola } \\
\text { tion }\end{array}$ & $\begin{array}{c}\text { Phase } \\
\text { Correction }\end{array}$ & FFT & Acquisition & Processing \\
\hline $\begin{array}{c}\text { SED } \\
850 \text { Band }\end{array}$ & 180 & 180 & 360 & $0.19 / 0.11$ & 0.17 & 0.88 & 0.045 & 1.80 & 1.40 \\
\hline $\begin{array}{c}\text { SED } \\
\text { Dual Band }\end{array}$ & 300 & 300 & 600 & $0.21 / 0.15$ & 0.28 & 1.26 & 0.057 & 3.00 & 1.96 \\
\hline $\begin{array}{l}\text { Spectral Line } \\
850 \text { Band }\end{array}$ & 180 & 3000 & 3180 & $0.27 / 1.04$ & 0.85 & 6.48 & 0.406 & 15.90 & 9.05 \\
\hline $\begin{array}{l}\text { Spectral Line } \\
\text { Dual Band }\end{array}$ & 300 & 5000 & 5300 & $0.36 / 1.75$ & 1.39 & 10.54 & 0.670 & 26.50 & 14.71 \\
\hline
\end{tabular}

\section{CONCLUSION}

The FTS-2 project completed the PDR stage in July 2005 and the CDR is scheduled for June 2006. Delivery of the instrument to the JCMT will occur in mid 2007, and commissioning will occur after the SCUBA-2 commissioning phase is complete.

\section{ACKNOWLEDGEMENTS}

On behalf of the Canadian SCUBA-2 consortium, the authors acknowledge the support of a CFI international access award for Canadian participation in the SCUBA-2 project. Thanks also go to Side by Side Optical Engineering (http://www.sidebysideoptics.com/) for their effort in the optical design. 


\section{REFERENCES}

1. P. Andre, D. Ward-Thompson and M. Barsony, "From Prestellar Cores to Protostars: the Initial Conditions of Star Formation," Protostars and Planets IV, U. Arizona Press, 59 (2000).

2. F. Shu, Z. Li and A. Allen, "Does Magnetic Levitation or Suspension Define the Masses of Forming Stars?", Ap. J. 601, 930-951 (2004).

3. W. S. Holland, E. I. Robson, W. K. Gear, C. R. Cunningham, J. F. Lightfoot, T. Jenness, R. J. Ivison, J. A. Stevens, P. A. R. Ade, M. J. Griffin, W. D. Duncan, J. A. Murphy and D. A. Naylor, "SCUBA: a common-user submillimetre camera operating on the James Clerk Maxwell Telescope," Mon. Not. R. Astron. Soc. 303, 659-672 (1999).

4. W. S. Holland, W. D. Duncan, B. D. Kelley, K. D. Irwin, A. J. Walton, P. A. R. Ade and E. I. Robson, "SCUBA-2: A large format submillimetre camera on the James Clerk Maxwell Telescope," Proc. SPIE, Millimeter and Submillimeter Detectors for Astronomy 4855, 1-18 (2003).

5. D. A. Naylor and B. G. Gom, "SCUBA-2 imaging Fourier transform spectrometer," Proc. SPIE, Imaging Spectrometry IX 5159, 91-101 (2004).

6. P. A. R. Ade, P. A. Hamilton and D. A. Naylor, “An Absolute Dual Beam Emission Spectrometer," Optical Society of America, FTS topical meeting poster FWE3, Santa Barbara, California, (1999).

7. B. G. Gom and D. A. Naylor, "An update on the imaging Fourier transform spectrometer for SCUBA-2," Proc. SPIE, Astronomical Telescopes and Instrumentation 5498 (2004).

8. Zaber Technologies Inc., http://ww.zaber.com/

9. D. A. Naylor and A. A. Schultz, "A Simple Technique for Accurately Measuring the Dihedral Angle of a Roof-Top Morror" Optics and Photonics News 11, issue 11, (2000).

10. Aertotech Inc., http://www.aerotech.com/

11. D. A. Naylor, T. R. Fulton, P. W. Davis, I. M. Chapman, B. G. Gom, L. D. Spencer, J. V. Lindner, N. E. NelsonFitzpatrick, M. K. Tahic and G. R. Davis, "Data processing pipeline for a time-sampled imaging Fourier transform spectrometer," Proc. SPIE, Imaging Spectrometry X 5546, (2004).

12. R. S. Le Poole and H. W. van Someren Greve, "DREAM, the Dutch REal-time Acquisition Mode for SCUBA", http://www.strw.leidenuniv.nl/ lepoole/ (1998).

13. D. A. Naylor, B. G. Gom, I. S. Schofield, G. J. Tompkins and G. R. Davis, "Mach-Zehnder Fourier transform spectrometer for astronomical spectroscopy at submillimeter wavelengths," Proc. SPIE, Millimeter and Submillimeter Detectors for Astronomy 4855, 540-551 (2003).

14. T. Jenness, F. Economou, D. Scott, D. Kelly and W. S. Holland, "Preliminary Design of the SCUBA-2 Data Reduction Pipeline," ADASS XIII, (2004).

15. T. J. Farrell, K. Shortridge and J. A. Bailey, "DRAMA: An Environment for Instrumentation Software," Bulletin of the $A A S, 25(2),(1993)$.

16. A. G. Gibb, D. Scott, T. Jenness, F. Economou, B. D. Kelly and W. S. Holland, "Design of the SCUBA-2 Quick Look Display and Data Reduction Pipeline," ASP, Astronomical Data Analysis Software and Systems XIV, 347, 585 (2005).

17. Paul N. Swarztrauber, http://www.netlib.org/fftpack/

18. J. Hicklin, C. Moler, P. Webb, R. Boisvert et al., http://math.nist.gov/javanumerics/jama/

19. Star Java, http://www.starlink.ac.uk/java/java.htm

20. N. P. Rees, F. Economou, T. Jenness, R. D. Kackley, C. A. Walther, W. R. Dent, M. Folger, X. Gao, D. Kelly, J. F. Lightfoot, I. Pain, G. Hovey and R. O. Redman, "JCMT observatory control system," Proc. SPIE, Advanced Telescope and Instrumentation Control Software II 4848, (2002).

21. B. D. Kelly, http://www.roe.ac.uk/ukatc/projects/scubatwo/documents/software/sc2-sof-s200-026.pdf

22. http://www.rtai.org/ 